\title{
Indocyanine green guided liver resection for hepatocellular carcinoma - literature review
}

\author{
Nicolae Bacalbasa ${ }^{1,2,3}$, Irina Balescu ${ }^{4}$, Camelia C. Diaconu ${ }^{1,5}$, Laura lliescu ${ }^{1,6}$, \\ Mihaela Vilcu ${ }^{1,7}$, Lucian Pop ${ }^{8}$, Mihai Dimitriu ${ }^{1,9}$, Cristian Balalau ${ }^{1,10}$, Alexandru Filipescu ${ }^{1,11}$, \\ Carmen Savu' ${ }^{12}$, Cornel Savu ${ }^{1,13}$, Emil Belu' ${ }^{14}$, Iulian Brezean ${ }^{1,7}$ \\ 1"Carol Davila" University of Medicine and Pharmacy, Bucharest, Romania \\ 2Department of Obstetrics and Gynecology, "I. Cantacuzino" Clinical Hospital, Bucharest, Romania \\ ${ }^{3}$ Department of Visceral Surgery, Center of Excellence in Translational Medicine \\ Fundeni Clinical Institute, Bucharest, Romania \\ ${ }^{4}$ Department of Surgery, Ponderas Academic Hospital, Bucharest, Romania \\ ${ }^{5}$ Department of Internal Medicine, University Emergency Hospital, Bucharest, Romania \\ ${ }^{6}$ Department of Internal Medicine, Fundeni Clinical Institute, Bucharest, Romania \\ ${ }^{7}$ Department of Surgery, "I. Cantacuzino" Clinical Hospital, Bucharest, Romania \\ 8"Alessandrescu-Rusescu" National Institute of Mother and Child Health, \\ Fetal Medicine Excellence Research Center, Bucharest, Romania \\ ${ }^{9}$ Department of Obstetrics and Gynecology, \\ "Sf. Pantelimon" Emergency Clinical Hospital, Bucharest, Romania \\ ${ }^{10}$ Department of Surgery, Pantelimon Clinical Hospital, Bucharest, Romania \\ ${ }^{11}$ Department of Obstetrics and Gynecology, Elias Emergency Hospital, Bucharest, Romania \\ ${ }^{12}$ Department of Anesthesiology, Fundeni Clinical Institute, Bucharest, Romania \\ ${ }^{13}$ Department of Thoracic Surgery, "Marius Nasta" Institute of Pneumonology, Bucharest, Romania \\ ${ }^{14}$ Department of Oncology, "Prof. Dr. Al. Trestioreanu” Institute of Oncology, Bucharest, Romania
}

\begin{abstract}
Anatomical liver resection guided by segmental portal inflow compression proved to be an efficient tool in order to maximize the curative surgical treatment in hepatocellular carcinoma. In this respect, in the last decades, once the concept of indocyanine guided surgery gained more field, the method has been also implemented in hepatic surgery. Therefore it seems that intravenous injection followed by portal pedicle clamping and liver inspection in infrared light inspection might delimitate the segments of the liver and might successfully guide the gesture of anatomical liver resection. The current paper is a literature review of the studies which analyzed the efficacy and safety of indocyanine green guided liver surgery.
\end{abstract}

Keywords: indocyanine green, anatomical liver resection, infrared

Abbreviations: ICG = Indocyanine green

\section{INTRODUCTION}

Hepatocellular carcinoma represents a common malignancy affecting people worldwide which proves to be efficiently treated by therapeutic meanings such as resection or radiofrequency ablation $(1,2)$. Meanwhile, several studies came to demonstrate the superiority of surgery in front of radiofrequency ablation in cases presenting unique lesions (2). In this respect attention was focused on improving the surgical technique in order to maximize the effectiveness of the procedure and to increase the lifespan in such cases. 


\section{ANATOMICAL VERSUS NON-ANATOMICAL LIVER RESECTION IN HEPATOCELLULAR CARCINOMA}

Although initially it has been considered that non-anatomical resections with negative resection margins should be the option of choice in patients with unique hepatocellular carcinoma, further studies came to demonstrate that these patients are predisposed on developing further metastases, frequently located at the level of the same portal territory. In this respect, certain studies underlined the necessity of performing anatomical resections in order to resect the entire portal territory and therefore to prevent the process of intrahepatic dissemination via portal vein $(3,4)$. The procedure has been initially proposed by Makuuchi et al. and proved to significantly improve the long term outcomes of patients with hepatocellular carcinoma $(5,6)$. In order to achieve anatomical resections, certain authors proposed ICG injection followed by fluorescence imaging with encouraging results (7).

\section{ROLE OF INDOCYANINE GUIDED SURGERY FOR ANATOMICAL LIVER RESECTION IN PATIENTS WITH HEPATOCELLULAR CARCINOMA}

In the last decade increasing evidence came to sustain the efficacy of indocyanine green utility in order to improve the information regarding the vascularization of a certain territory, to establish the viability of a certain viscera or to verify the feasibility of performing a certain anastomosis. Furthermore, the utility of indocyanine green injection followed by infrared inspection was also studied in patients considered as candidates for anatomical resections. The method consists of intravenous injection of indocyanine green clamping the afferent portal pedicle and infrared visualization of the liver parenchyma (7). Meanwhile, certain authors proposed ICG administration within two weeks before surgery in order to study the functional reserve of the liver parenchyma, intraoperatively, followed by portal pedicle clamping in order to guide the resection and intraoperatively but at the level of the main biliary duct in order to detect and bile leakage (7).

The method has been recently proposed and investigated in cases in which laparoscopic approach is attempted due to the fact that the minimally invasive technique is more demanding when compared to the open technique when it comes to portal staining and inflow portal clamping (8). The method therefore might be performed in two manners: the first one provides a negative staining and consists of identification of the correspondent portal pedicle which is further clamped and ICG is injected intravenously; therefore, the whole parenchyma excepting the area correspondent to the afferent portal pedicle will present ICG positive staining; furthermore, only the area of negative staining will be resected. The second manner consists of ultrasound guided portal puncture and ICG injection and therefore is considered as positive staining. However, the second option is considered to be more difficult to be performed in a minimally invasive manner due to the necessity of laparoscopic ultrasound in order to identify the corresponding portal pedicle; however, it should not be omitted the fact that negative staining also associates a degree of complex laparoscopic gestures in order to dissect and clamp the corresponding Glissonian pedicle (9-11). Moreover, the negative staining procedure also presents a higher risk of bile duct injury during Glissonian dissection and might be more demanding when performed for segment VII and VIII tumors (9).

One of the most interesting studies conducted on the theme of negative staining during laparoscopic surgery aiming anatomical liver resection was conducted by Urade et al. and was published in 2019 (8); in this study the authors introduced three cases submitted to laparoscopic resection for hepatocellular carcinoma - in two cases and respectively metastatic liver lesions from colorectal cancer in the third cases. In all cases negative staining was performed and consisted of intravenous injection of $2.5 \mathrm{mg}$ of ICG followed by Glissonian pedicle clamping; in all cases the procedure was successfully ended in a minimally invasive manner and consisted of segments V/VIII resection in the first case, segment III resection in the second case and respectively a right anterior sectionectomy in the third case (8).

In order to diminish the risks and inconveniences caused by the two methods presented in the paragraphs above, certain authors proposed another protocol of ICG injection, by using the percutaneous route; therefore, the Japanese authors conducted by Aoki et al. described their preliminary experience with percutaneous injection of ICG directly in the corresponding pedicle of the portal vein immediately before surgery (12). Furthermore, the principle of positive staining was applied; by using this method, the authors excluded the necessity of intraoperative ultrasound, a standard abdominal ultrasound being used instead. The method was successfully performed in 14 cases, in nine of them the 
indication for surgery being represented by hepatocellular carcinoma while in the remaining five cases liver metastases from colorectal cancer being the indication for surgery. In all cases an adequate identification of the tumor was provided while the resection margins were negative, demonstrating in this way the feasibility, efficacy and oncological safety of the procedure (12).

\section{ROLE OF INDOCYANINE GREEN INJECTION IN LIVER HARVESTING FROM LIVING DONOR}

Once the method of ICG guided anatomical liver resection has gained more popularity, the principle of the method was also implemented in order to facilitate liver harvesting in living donor for both adults and children $(13,14)$. Therefore, the method seems to be safe and effective in order to establish the anatomical boundaries and to further guide the laparoscopic harvesting. However, only isolated cases have been reported so far, the method not being part of the standard use for the present moment $(13,14)$.

\section{CONCLUSIONS}

ICG injection during laparoscopic approach seems to be a safe and effective method in order to guide the extent of resection and to provide complete, typical hepatic resections. In order to improve the outcomes, multiple variants of injection have been proposed, leading in this way to positive and negative staining. However, the method seems to be a demanding one, advanced surgical skills of laparoscopy being needed. Meanwhile, certain authors went even further and implemented the knowledge of ICG guided anatomical resections for hepatocellular carcinoma in the field of donor liver transplantation; in this way, a more rapid and safe harvesting of the liver lobe which will be transplanted seems to be obtained. However, all these methods have not been yet studied in large randomized studies, further work being still needed before creating a standard therapeutic protocol which might be based on these findings.

\section{Acknowledgements}

This work was supported by the project entitled „Multidisciplinary Consortium for Supporting the Research Skills in Diagnosing, Treating and Identifying Predictive Factors of Malignant Gynecologic Disorders", project number PN-III-P1-1.2-PCCDI2017-0833.

\section{Conflict of interest: none declared}

\section{REFERENCES}

1. Bosch FX, Ribes J, Diaz M, Cleries R. Primary liver cancer: Worldwide incidence and trends. Gastroenterology 2004;127:S5-S16.

2. Wang $Y$, Luo Q, Li Y et al. Radiofrequency ablation versus hepatic resection for small hepatocellular carcinomas: A meta-analysis of randomized and non-randomized controlled trials. PLoS One. 2014:9:e84484.

3. Shindoh J, Makuuchi M, Matsuyama Y et al. Complete removal of the tumor-bearing portal territory decreases local tumor recurrence and improves disease-specific survival of patients with hepatocellular carcinoma. J Hepatol. 2016;64:594-600.

4. Hasegawa K, Kokudo N, Imamura H et al. Prognostic impact of anatomic resection for hepatocellular carcinoma. Ann Surg. 2005 242:252-259.

5. Makuuchi M. Surgical treatment for HCC -- special reference to anatomical resection. Int J Surg. 2013;11(Suppl 1):S47-S49.

6. Makuuchi M, Mori T, Gunven P et al. Safety of hemihepatic vascular occlusion during resection of the liver. Surg Gynecol Obstet. 1987;164:155-158.

7. Otsuka $Y$, Katagiri T, Kubota $Y$ et al. Usefulness of ICG fluorescence imaging system in laparoscopic liver resection for liver tumors. HPB 2016;18(S1):e1-e384.

8. Urade T, Sawa H, Iwatani $\mathrm{Y}$ et al. Laparoscopic anatomical liver resection using indocyanine green fluorescence imaging. Asian $\mathrm{J}$ Surg. 2020:43:362-368.
9. Ishizawa T, Zuker NB, Kokudo N, Gayet B. Positive and negative staining of hepatic segments by use of fluorescent imaging techniques during laparoscopic hepatectomy. Arch Surg. 2012;147:393-394.

10. Sakoda M, Ueno S, lino S et al. Anatomical laparoscopic hepatectomy for hepatocellular carcinoma using indocyanine green fluorescence imaging. J Laparoendosc Adv Surg Tech. 2014;24:878-882.

11. Mizuno T, Sheth R, Yamamoto M et al. Laparoscopic Glissonean Pedicle Transection (Takasaki) for Negative Fluorescent Counterstaining of Segment 6. Ann Surg Oncol. 2017;24:1046-1047.

12. Aoki T, Koizumi T, Mansour DA et al. Ultrasound-Guided Preoperative Positive Percutaneous Indocyanine Green Fluorescence Staining for Laparoscopic Anatomical Liver Resection. J Am Coll Surg. 2020;230:e7-e12.

13. Suh KS, Hong SK, Lee KW et al. Pure laparoscopic living donor hepatectomy: Focus on 55 donors undergoing right hepatectomy. Am J Transplant. 2018;18:434-443.

14. Soubrane O, Cherqui D, Scatton O et al. Laparoscopic left lateral sectionectomy in living donors: safety and reproducibility of the technique in a single center. Ann Surg. 2006;244:815-820. 